\title{
Effects of Core Moieties on the Kinetics and Microstructures of Hyperbranched Polymers. Theoretical and Off-Lattice Monte Carlo Simulation Approach
}

\author{
Yong Uk LEE and Won Ho JO ${ }^{\dagger}$ \\ Hyperstructured Organic Materials Research Center and School of Materials Science and Engineering, \\ Seoul National University, Seoul 151-742, Korea
}

(Received May 10, 2002; Accepted August 8, 2002)

\begin{abstract}
Kinetic equations describing the conversion $\left(p_{\mathrm{A}}\right)$ and number average degree of polymerization $\left(\mathrm{DP}_{\mathrm{n}}\right)$ as a function of time for polymerization of $\mathrm{AB}_{n}$ type monomers with $\mathrm{B}_{\mathrm{f}}$ type core molecules were derived under polycondensation and multibranching polymerization (MBP) conditions. An off-lattice Monte Carlo simulation was performed to analyze polymerization under MBP and compare simulated results with theoretical predictions. The theoretical equations predict that the conversion for MBP shows a S-type curve whereas the curve for polycondensation shows a typical shape similar to that for homopolycondensation of $\mathrm{AB}_{n}$ type monomers without a core molecule. For both cases, $\mathrm{DP}_{\mathrm{n}}$ is controlled by the ratio $r$ of the number of core molecules to that of monomers. Simulation results show that the conversion curve also shows a S-type curve, this being consistent with theoretical prediction. From simulation, $\mathrm{DP}_{\mathrm{n}}$ can be controlled simply by varying the concentration of core molecules, as predicted by the theory. Low core functionality and low core concentration increase the degree of branching of the hyperbranched polymers.

KEY WORDS Kinetics / Microstructure / Core Moiety / Hyperbranched Polymer / Monte Carlo Simulation /
\end{abstract}

Hyperbranched polymers obtained from $\mathrm{AB}_{n}$ type monomers have recently attracted much interest, ${ }^{1,2}$ because they have unique properties, such as high functionality and low viscosity, and can be polymerized in a simple way compared to the tedious repetitive synthetic routes of dendrimers. As predicted by Flory, ${ }^{3}$ however, hyperbranched polymers have extremely broad molecular weight distribution at reasonably high molecular weight or high conversion. The branched structure of hyperbranched polymers is not perfect compared to dendrimers.

Control of the degree of polymerization, polydispersity index, and degree of branching, ${ }^{4}$ is very important to obtain hyperbranched polymers with demanding properties. For this purpose, the use of $B_{f}$ type core molecules was suggested for polymerization of $\mathrm{AB}_{n}$ type monomers. ${ }^{5,6}$ The first synthetic approach was polymerization of 2,2-bis(hydroxymethyl) propionic acid $\left(\mathrm{AB}_{2}\right.$ type monomer) with 2-ethyl-2(hydroxymethyl)-1,3propanediol ( $\mathrm{B}_{3}$ type core molecule) by a pseudo one-step reaction where stoichiometric amounts of monomers corresponding to each generation were added successively. ${ }^{6}$ Molecular weight distribution of hyperbranched polymers under polycondensation in the presence of a multifunctional core molecule was predicted theoretically by Yan et $a l^{7,8}$ The addition of small amounts of core molecules leads to a marked re- duction in the polydispersity index of hyperbranched polymers. Palladium-catalyzed ring-opening polymerization of cyclic carbamates has been designated as "multibranching polymerization (MBP)" by Suzuki et $a l .{ }^{10,11}$ In MBP, the reaction starts from an initiator. The number of active sites increases as polymerization proceeds and consequently a hyperbranched polymer containing an initiator as a core moiety is produced. Thus, the structure of a hyperbranched polymer can be easily defined by initiator content added, and the molecular weight of the polymer is controlled by the ratio of initiator to monomer. When a macro-initiator is used, a graft or block copolymer with a part of hyperbranched polymer can be prepared. However, the kinetics of MBP and the microstructure of the polymers have not been studied, although some theoretical approaches on kinetics of homopolymerization of $\mathrm{AB}_{n}$ type monomers have been reported by Beginn et al. ${ }^{15}$

In previous studies, ${ }^{12,13}$ polycondensation of $\mathrm{AB}_{2}$ type monomers was simulated using an off-lattice Monte Carlo method to investigate the polymerization kinetics and microstructures of hyperbranched polymers prepared under different reaction conditions. In this study, kinetic equations are derived theoretically in terms of conversion and degree of polymerization for polycondensation of $\mathrm{AB}_{n}$ type monomers in the presence of $\mathrm{B}_{\mathrm{f}}$ type core molecules under MBP and offlattice Monte Carlo simulation was performed to an- 
alyze polymerization under MBP and compare simulated results with theoretical predictions.

\section{MODEL AND SIMULATIONS}

Off-lattice Monte Carlo simulation was performed on a $30 \sigma \times 30 \sigma \times 30 \sigma$ cubic cell $(\sigma$, diameter of a segment and set at 1.0) with periodic boundary conditions in $x, y$, and $z$ directions. $\mathrm{An} \mathrm{AB}_{n}$ type monomer consists of 1 arm containing an A functional group and $n$ arms containing $\mathrm{B}$ functional groups. $\mathrm{A}_{\mathrm{f}}$ core molecule consists of $f$ arms with each arm containing a B functional group. For both monomer and core molecules, each arm possesses one spacer segment. The cell was filled with 1000 monomers and $1000 \times r$ core molecules, where $r$ is the ratio of core molecules to monomers. For example, if $r=0.01,1000$ monomers and 10 core molecules were used in the simulation. Simulation was performed five times under each simulation condition, and the average is reported. A harmonic type potential and shifted Lennard-Jones potential were used for bonded and non-bonded interactions between segments, respectively. Details of the simulation are described in our previous papers. ${ }^{12,13}$

Since MBP is simulated here, the core molecule acts as initiator so that the monomer reacts only with polymers containing a core moiety, and therefore condensation reactions between polymers or between monomers cannot occur. Only B functional groups incorporated in growing polymers have reactivity. This kind of reaction was first reported by Suzuki ${ }^{10,11}$ and recently extended to the polymerization of glycidol monomers under slow monomer addition. ${ }^{14}$ During simulation, an A functional group reacts only with a B functional group and vice versa, and depolymerization and cyclization reactions do not occur.

Conversion $\left(p_{\mathrm{A}}\right)$ is defined as the number fractions of A functional groups reacted. The number average degree of polymerization $\left(\mathrm{DP}_{\mathrm{n}}\right)$ and polydispersity index $(P I)$ were calculated as functions of $p_{\mathrm{A}}$. The degree of branching (DB) and average number of branches $(A N B)$ were calculated from the fractions of dendritic $(D)$, linear $(L)$, and terminal unit $(T)$.

\section{DERIVATION OF KINETIC EQUATIONS}

\section{Case I. Polycondensation of AB $B_{n}$ Type Monomers}

As the starting point for further derivations for cases II and III, we briefly introduce kinetic equations for polycondensation of $\mathrm{AB}_{n}$ type monomers, as derived by Beginn et al. ${ }^{15}$ When the reaction is given as $\mathrm{A}+\mathrm{B} \stackrel{k_{n}}{\longrightarrow} \mathrm{AB}$, where $k_{n}$ is a reactivity constant, the rate of consumption of $\mathrm{A}$ functional groups is

$$
-\frac{\mathrm{d}[\mathrm{A}]}{\mathrm{d} t}=k_{n}[\mathrm{~A}][\mathrm{B}]
$$

where $[\mathrm{A}]$ and $[\mathrm{B}]$ are concentrations of $\mathrm{A}$ and $\mathrm{B}$ functional groups, respectively. Assuming that (i) the reactivity of all groups is identical, (ii) the reaction is not catalyzed by one of the functional groups A or B, (iii) steric hindrance is not taken into account during reaction, and (iv) cyclization is not allowed, the conversion of A functional groups $\left(p_{\mathrm{A}}\right)$ and number average degree of polymerization $\left(\mathrm{DP}_{\mathrm{n}}\right)$ as a function of time are derived, respectively, as eqs 2 and 3:

$$
\begin{aligned}
& p_{\mathrm{A}}=\frac{1-\exp \left\{-(n-1) k_{n}[\mathrm{~A}]_{0} t\right\}}{1-\frac{1}{n} \exp \left\{-(n-1) k_{n}[\mathrm{~A}]_{0} t\right\}} \\
& \mathrm{DP}_{\mathrm{n}}=\frac{[\mathrm{A}]_{0}}{[\mathrm{~A}]}=\frac{1}{1-p_{\mathrm{A}}}=\frac{n-\exp \left\{-(n-1) k_{n}[\mathrm{~A}]_{0} t\right\}}{(n-1) \exp \left\{-(n-1) k_{n}[\mathrm{~A}]_{0} t\right\}}
\end{aligned}
$$

where $[\mathrm{A}]_{0}$ is the initial concentration of A functional group.

Case II. Polycondensation of $A B_{n}$ Type Monomers with $B_{f}$ Type Core Molecules

When $\mathrm{B}_{f}$ type core molecules are introduced in polycondensation of $\mathrm{AB}_{n}$ type monomers, kinetic equations as expressed by $p_{\mathrm{A}}$ and $\mathrm{DP}_{\mathrm{n}}$ as a function of time may be derived in the same way as in case I under the same assumptions. The concentration of B functional groups $[B]$ is expressed as the sum of $B$ functional groups from core molecules and those from monomers, i.e.,

$$
[\mathrm{B}]=\left[\mathrm{B}_{\mathrm{f}}\right]_{0} f+(n-1)[\mathrm{A}]_{0}+[\mathrm{A}]
$$

where $\left[\mathrm{B}_{f}\right]_{0}=r[\mathrm{~A}]_{0}$ is the initial concentration of $\mathrm{B}_{f}$ type core molecules. The first term of the right hand side of eq 4 represents the number of $B$ functional groups from core molecules. Integrating eq 1 after substituting eq 4 into eq 1 leads to

$$
\begin{aligned}
\frac{1}{(r f+n-1)[\mathrm{A}]_{0}}\left\{\ln \left[\frac{[\mathrm{A}]}{[\mathrm{A}]_{0}}\right]\right. \\
\left.-\ln \left[\frac{[\mathrm{A}]+(r f+n-1)[\mathrm{A}]_{0}}{[\mathrm{~A}]_{0}+(r f+n-1)[A]_{0}}\right]\right\}=-k_{n} t
\end{aligned}
$$

Rearrangement of eq 5 gives an expression for the concentration of A functional group as a function of time as follows:

$$
[\mathrm{A}]=\frac{(r f+n-1)[\mathrm{A}]_{0} \exp \left\{-k_{n} t(r f+n-1)[\mathrm{A}]_{0}\right\}}{1+(r f+n-1)-\exp \left\{-k_{n} t(r f+n-1)[\mathrm{A}]_{0}\right\}}
$$


Conversion and degree of polymerization as a function of time may be obtained from eq 6 as follows:

$$
\begin{aligned}
& p_{\mathrm{A}}=\frac{[\mathrm{A}]_{0}-[\mathrm{A}]}{[\mathrm{A}]_{0}}=\frac{1-\exp \left\{-(r f+n-1) k_{n}[\mathrm{~A}]_{0} t\right\}}{1-\frac{1}{r f+n} \exp \left\{-(r f+n-1) k_{n}[\mathrm{~A}]_{0} t\right\}} \\
& \mathrm{DP}_{n}=\frac{\left[\mathrm{B}_{f}\right]_{0}+[\mathrm{A}]_{0}}{\left[\mathrm{~B}_{f}\right]_{0}+[\mathrm{A}]}=\frac{r+1}{r+1-p_{\mathrm{A}}} \\
& =\frac{(r f+n)(r+1)-\exp \left\{-(r f+n-1) k_{n}[\mathrm{~A}]_{0} t\right\}}{r(r f+n)+(r f+n-1-r) \exp \left\{-(r f+n-1) k_{n}[\mathrm{~A}]_{0} t\right\}}
\end{aligned}
$$

Case III. Multibranching Polymerization (MBP) of $A B_{n}$ Type Monomers with $B_{f}$ Type Core Molecules

Equation 1 is used as the starting point with the first three assumptions of case I. Cyclization does not occur because only reactions between monomer and growing polymer are taken into account in MBP mechanism. The concentration of $\mathrm{B}$ functional group $[\mathrm{B}]$ is given as the sum of B functional groups from core molecules and those from monomers incorporated in polymers:

$$
[\mathrm{B}]=\left[\mathrm{B}_{f}\right]_{0} f+(n-1)\left([\mathrm{A}]_{0}-[\mathrm{A}]\right)
$$

Integrating eq 1 with the aid of eq 9 gives

$$
\begin{aligned}
& \frac{1}{(r f+n-1)[\mathrm{A}]_{0}} \\
& \quad \times \ln \left[\frac{[\mathrm{A}]\left\{(r f+n-1)[\mathrm{A}]_{0}-(n-1)[\mathrm{A}]_{0}\right\}}{\left\{(r f+n-1)[\mathrm{A}]_{0}-(n-1)[\mathrm{A}]\right\}[\mathrm{A}]_{0}}\right]=-k_{n} t
\end{aligned}
$$

The concentration of A functional group is expressed as a function of time as,

$$
[\mathrm{A}]=\frac{(r f+n-1)[\mathrm{A}]_{0} \exp \left\{-k_{n} t(r f+n-1)[\mathrm{A}]_{0}\right\}}{r f+(n-1) \exp \left\{-k_{n} t(r f+n-1)[\mathrm{A}]_{0}\right\}}
$$

Rearranging eq 11 yields expressions for conversion and degree of polymerization as given by following two equations:

$$
\begin{aligned}
p_{\mathrm{A}} & =\frac{[\mathrm{A}]_{0}-[\mathrm{A}]}{[\mathrm{A}]_{0}} \\
& =\frac{1-\exp \left\{-k_{n} t(r f+n-1)[\mathrm{A}]_{0}\right\}}{1+\frac{1}{r f}(n-1) \exp \left\{-k_{n} t(r f+n-1)[\mathrm{A}]_{0}\right\}} \\
\mathrm{DP}_{n} & =\frac{\left[\mathrm{B}_{f}\right]_{0}+[\mathrm{A}]_{0}-[\mathrm{A}]}{\left[\mathrm{B}_{f}\right]_{0}}=1+\frac{1}{r} p_{\mathrm{A}} \\
& =1+\frac{1}{r} \times\left(\frac{1-\exp \left(-k_{n} t(r f+n-1)[\mathrm{A}]_{0}\right)}{1+\frac{1}{r f}(n-1) \exp \left(-k_{n} t(r f+n-1)[\mathrm{A}]_{0}\right)}\right)
\end{aligned}
$$
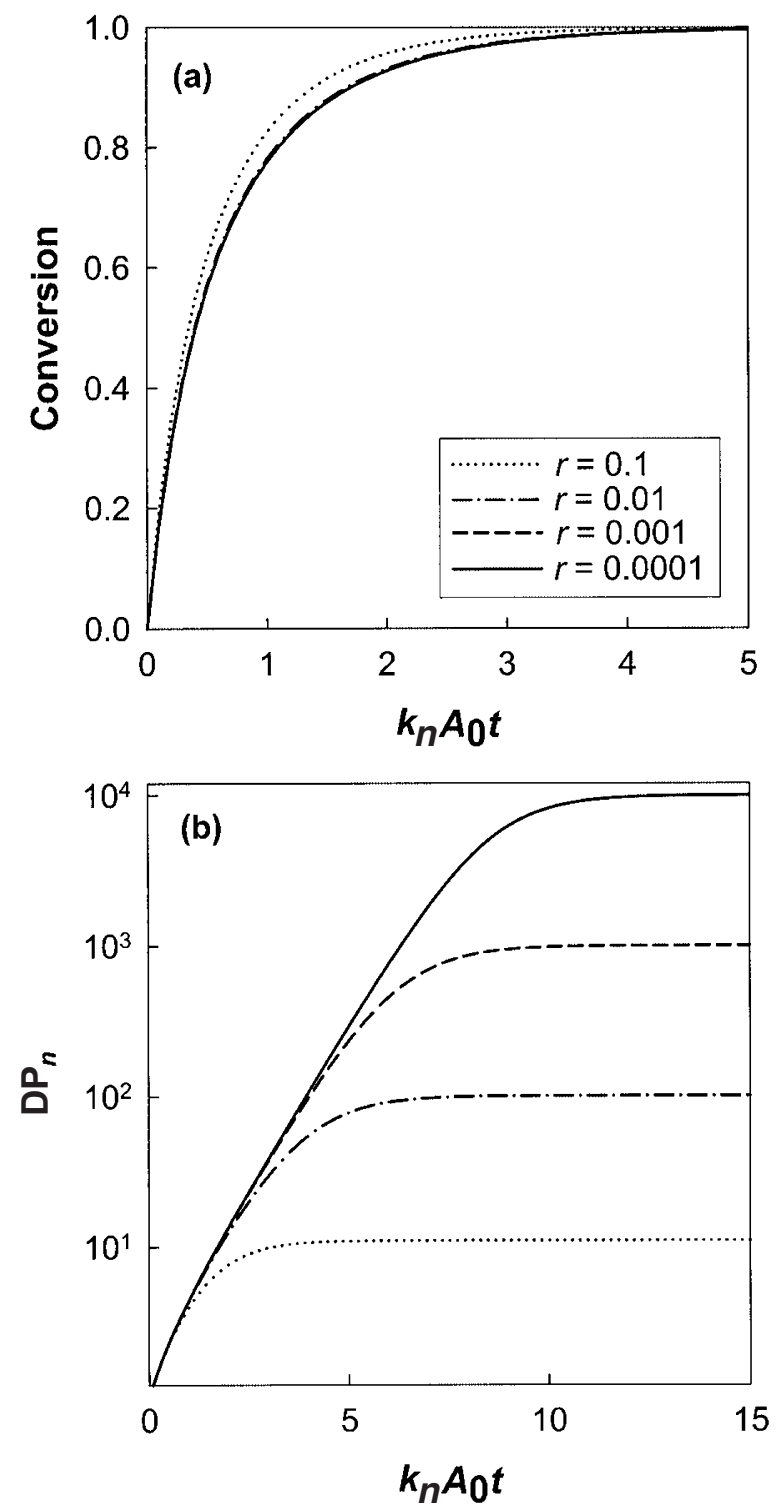

Figure 1. Theoretical predictions of (a) conversion and (b) number average degree of polymerization $\left(\mathrm{DP}_{\mathrm{n}}\right)$ as functions of time at various $r$ of core to monomer for case II, where $\mathrm{AB}_{2}$ monomers are polymerized in the presence of $\mathrm{B}_{3}$ core.

\section{RESULTS AND DISCUSSION}

The effects of the ratio $r$ on the conversion and $\mathrm{DP}_{\mathrm{n}}$ for polycondensation of $\mathrm{AB}_{n}$ type monomers with $\mathrm{B}_{f}$ type core molecules (case II) are shown in Figure 1. The addition of core molecules does not change the shape of the conversion curve significantly, as shown in Figure $1 \mathrm{a}$. The shape resembles that of homopolycondensation of $\mathrm{AB}_{n}$ type monomers (case I), because the reaction mechanism for both cases is basically polycondensation. $\mathrm{DP}_{\mathrm{n}}$ is strongly dependent on molecules added, i.e., $\mathrm{DP}_{\mathrm{n}}$ increases initially and converges to equilibrium, as conversion increases (Figure $1 \mathrm{~b}$ ). At the initial stage of polymerization, two hyperbranched polymers are produced: a polymer with a core, and one 

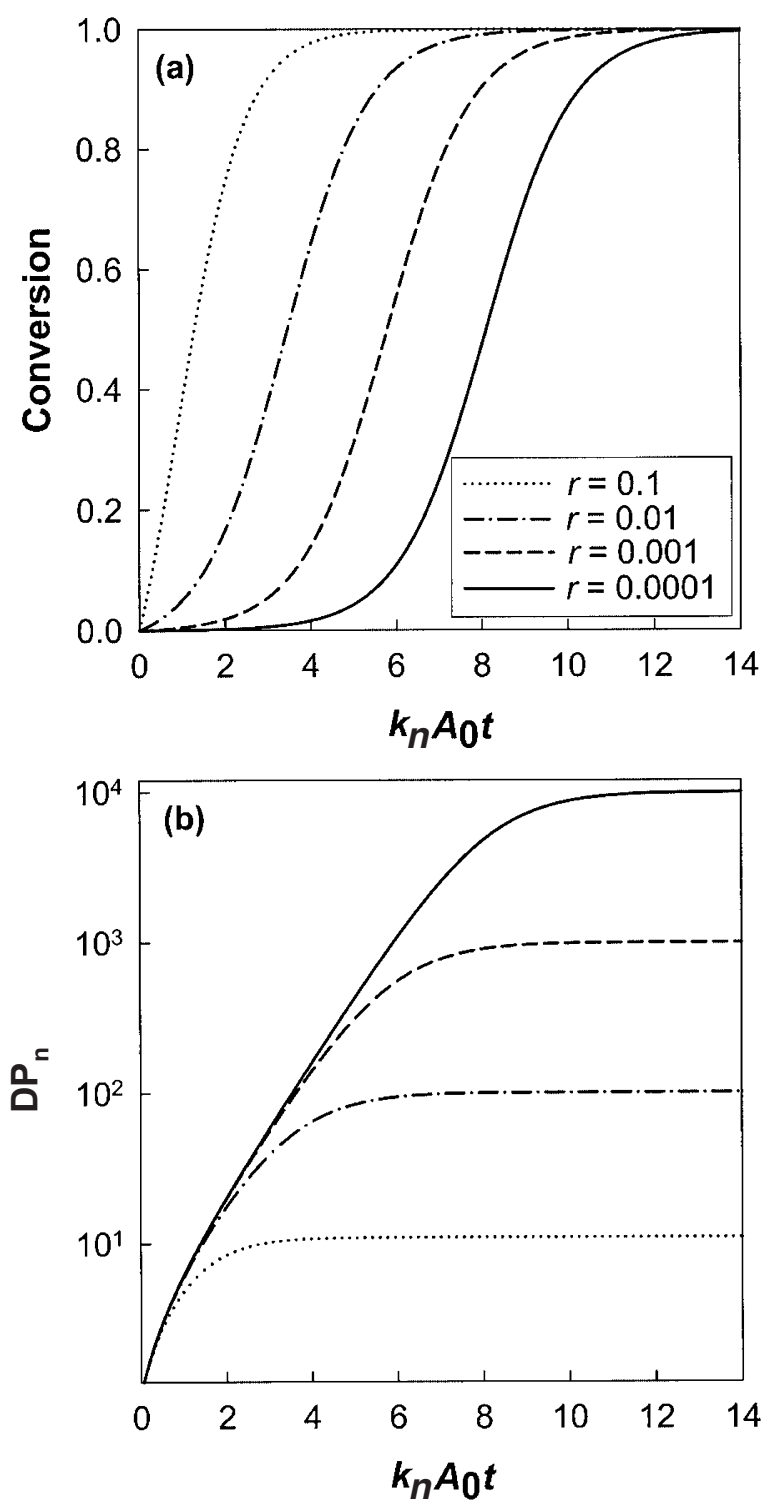

Figure 2. Theoretical predictions of (a) conversion and (b) number average degree of polymerization $\left(\mathrm{DP}_{\mathrm{n}}\right)$ as functions of time at various $r$ of core to monomer for case III, where $\mathrm{AB}_{2}$ monomers are polymerized in the presence of $\mathrm{B}_{3}$ core.

without a core. At the final stage of reaction, only polymers with a core moiety are present, and therefore $r$ becomes a crucial factor in determining $\mathrm{DP}_{\mathrm{n}}$, as evident from eq 8 with $p_{\mathrm{A}} \rightarrow 1$.

The kinetic behavior for case III is different from cases I and II, as evident from comparison of Figure 1 with Figure 2. In case III, the reaction starts from core molecules, and monomers can react only with growing polymers. Only a small amount of active B functional groups is thus available at the initial stage of reaction. As the reaction proceeds, the number of active B functional groups increases rapidly, and the reaction rate becomes faster as seen from increase of the slope in Figure 2 a. At high conversion, available monomers diminish and the reaction rate becomes slower. At high concentration of core molecules (e.g., $r=0.1$ in Fig-
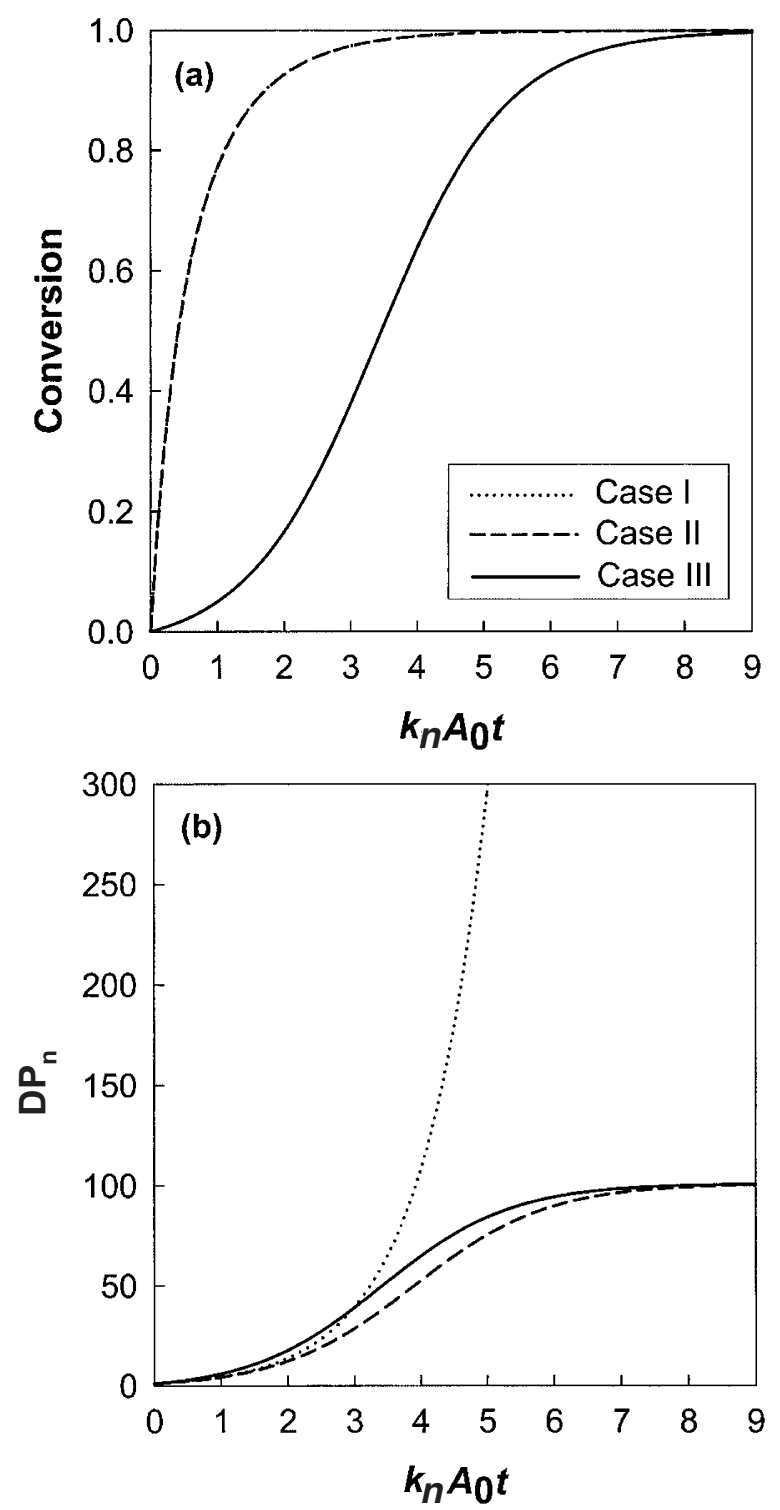

Figure 3. Comparison of theoretical predictions of (a) conversion and (b) number average degree of polymerization $\left(\mathrm{DP}_{\mathrm{n}}\right)$ of $\mathrm{AB}_{2}$ monomers with $\mathrm{B}_{3}$ core for cases I, II, and III at $r=0.01$.

ure $2 \mathrm{a}$ ), active B functional groups are enough so that conversion increases rapidly even at low conversion. The dependency of $\mathrm{DP}_{\mathrm{n}}$ on reaction time for case III (Figure $1 b$ ) is the same for the case II (Figure $2 b$ ).

Conversions and $\mathrm{DP}_{\mathrm{n}}$ for the three cases are compared in Figure 3. Conversions for the cases I and II show nearly the same behavior whereas conversion for case III is different. $\mathrm{DP}_{\mathrm{n}}$ for case II shows nearly the same behavior as case I at the initial stage, but finally converges to equilibrium for case III at the final stage of reaction, as shown in Figure $3 \mathrm{~b}$.

Theoretical predictions for the degree of branching (DB) and average number of branches $(A N B)$ for $\mathrm{AB}_{n}$ system under slow monomer addition (SMA) condition are suggested as follows: ${ }^{5}$

$$
\mathrm{DB}=\frac{n}{2 n-1}
$$



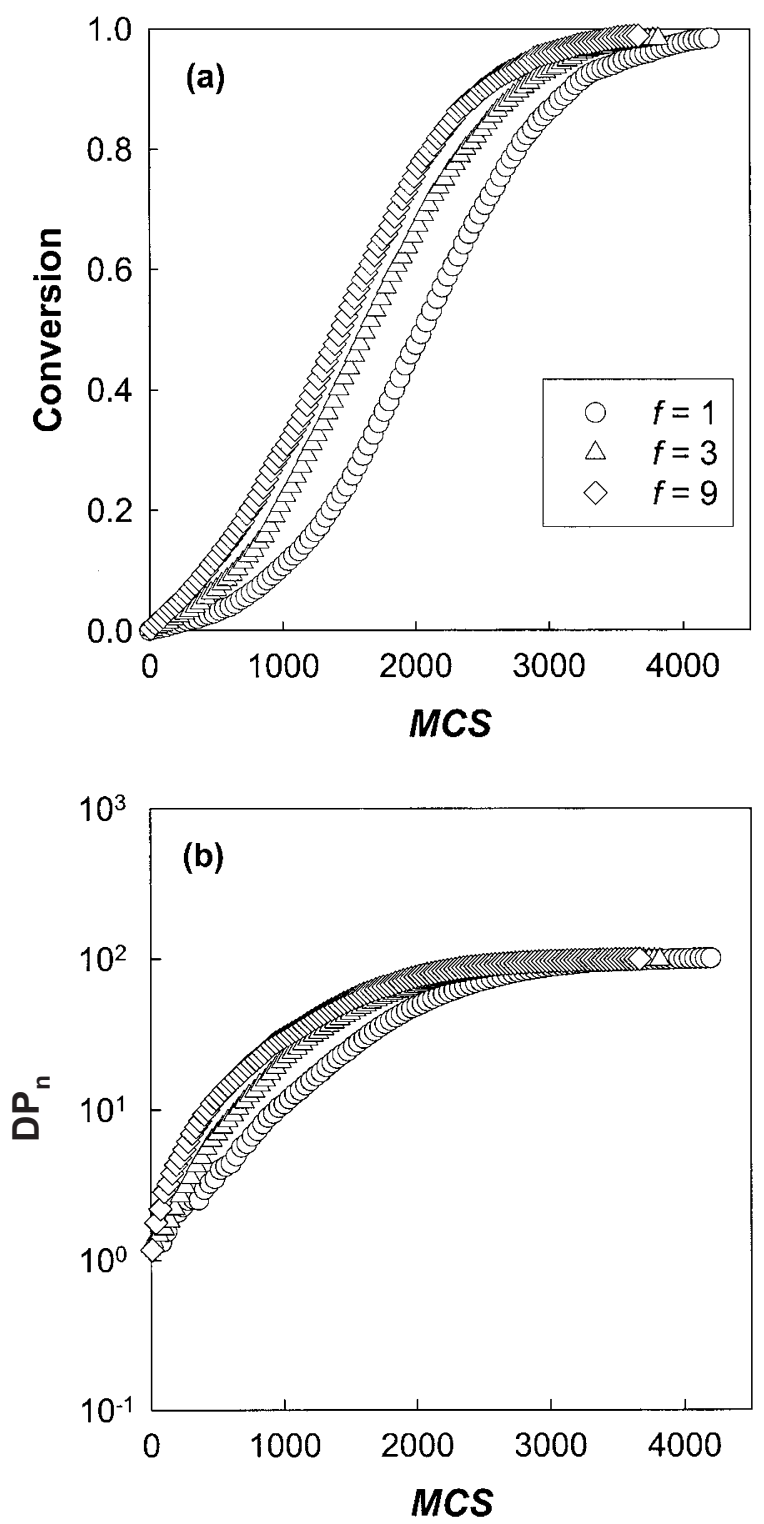

Figure 4. (a) Conversion and (b) number average degree of polymerization $\left(\mathrm{DP}_{\mathrm{n}}\right)$ of $\mathrm{AB}_{2}$ monomers from simulation performed under MBP at various core functionalities $(f)$ at $r=0.01$.

$$
A N B=\frac{n-1}{n}
$$

Off-lattice Monte Carlo simulation was performed under multibranching polymerization (MBP). Equations 14 and 15 can be used to analyze our simulation data, because the reaction conditions of MBP are nearly the same as for SMA. Figure 4 shows plots of simulated conversions and $\mathrm{DP}_{\mathrm{n}}$ against Monte Carlo step (MCS). $M C S$ is implicitly regarded as reaction time. The overall shape of conversion from simulation is an S-type (Figure 4 a), which is consistent with theoretical prediction (eq 12 and Figure $2 \mathrm{a}$ ). The overall shape and the value of $\mathrm{DP}_{\mathrm{n}}$ from simulation (Figure $4 \mathrm{~b}$ ) are nearly the same as theoretical predictions (eq 13 and Figure $2 b$ ). The number of initiating sites in simulation at the initial stage is proportional to $r \times f$ and therefore the conver-

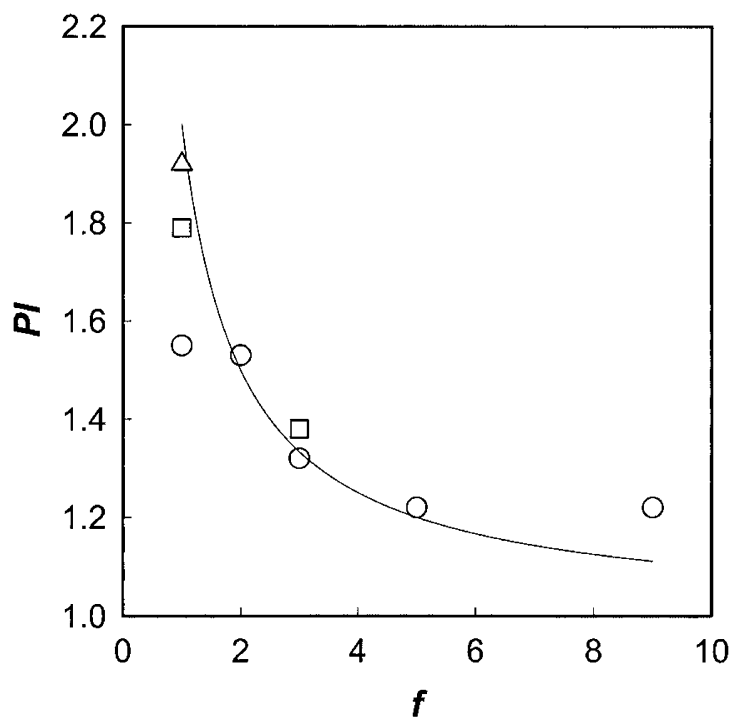

Figure 5. Polydispersity index $(P I)$ from simulation as functions of various core functionalities $f$ at $r=0.01(\bigcirc), 0.03(\square)$, and $0.09(\triangle)$, where $\mathrm{AB}_{2}$ type monomers are polymerized up to $p_{\mathrm{A}}=0.98$ under MBP.Solid line represents theoretical predictions from eq 16.

sion rate increases with core functionality $f$ at given $r$, as shown in Figure $4 \mathrm{a}$.

The effects of core functionality on the polydispersity $(P I)$ is shown in Figure 5. As discussed in the numerical works on polycondensation of $A B$ type monomers ${ }^{16}$ and slow monomer addition of $\mathrm{AB}_{n}$ type monomers, ${ }^{9}$ the effects of core functionality on $P I$ can be described by eq 16, derived for formation of star polymers:

$$
P I=1+\frac{1}{f}
$$

Simulated results were well consistent with theoretical ones. Some deviation of simulated results from the theoretical prediction was observed at high $(f=9)$ and low core functionality with low core concentration $(f=1$ and $r=0.01)$. This may arise from steric hindrance, not taken into account in theoretical studies.., 16 All unreacted functional groups are assumed to have the same probability of reaction in theoretical calculation, whereas in molecular simulation reaction probability of a functional group depends upon chemical environment. The B functional group located at the interior part of the polymer may have less chance to react with A functional groups than that located at exterior part, because the B functional group in the interior part is surrounded by nonreactive groups such as spacer or other groups. In this way, the steric effect can be incorporated in molecular simulation.

When $\mathrm{AB}_{2}$ type monomers are polymerized under SMA, the dendritic (D), linear (L) and terminal unit (T) ratio is predicted theoretically as $1: 1: 1$. The fraction of linear unit for SMA is smaller compared with poly- 

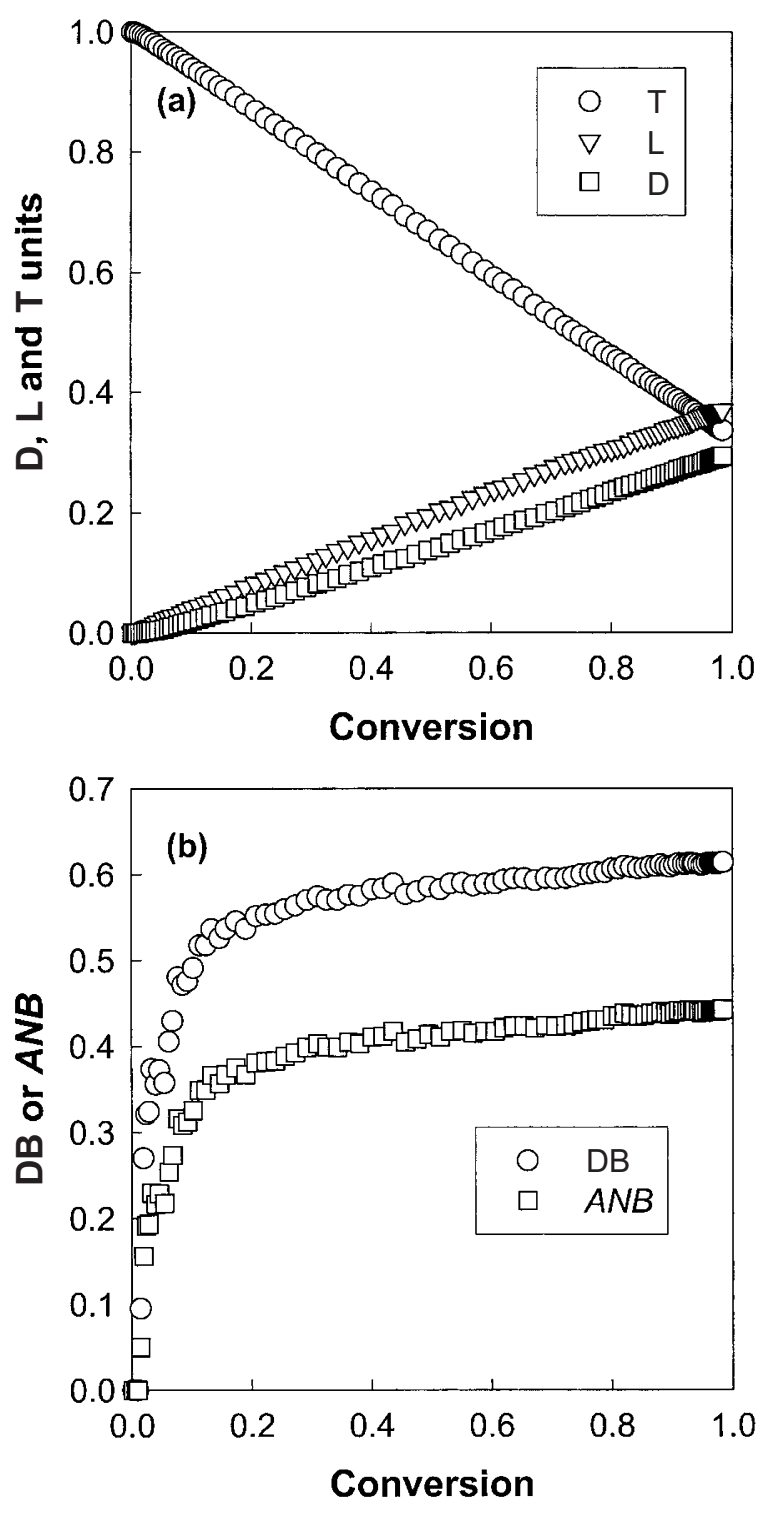

Figure 6. Plots of (a) fraction of dendritic (D), linear (L), and terminal unit (T), and (b) DB and $A N B$ vs. conversion, when $\mathrm{AB}_{2}$ monomers are polymerized under MBP in the presence of $\mathrm{B}_{3}$ core at $r=0.01$.

condensation of $\mathrm{AB}_{2}$ type monomers, where $\mathrm{D}: \mathrm{L}: \mathrm{T}=$ $1: 2: 1$. Conversion dependence of structural units is shown in Figure 6a, where the D, L, and T unit ratio at $p_{\mathrm{A}}=0.98$ is $0.29: 0.37: 0.34$. The simulated dendritic unit is slightly different from the values predicted theoretically. The lower dendritic units may arise from steric hindrance in simulation, whereas the theory does not take into account the steric hindrance. From structural units, DB and $A N B$ were calculated as 0.61 and 0.44 , respectively, values lower than theoretical 0.67 and 0.5 , respectively.

Figure 7 shows functionality dependence of structural units. As core functionality increases, the fraction of dendritic units decreases and therefore DB and $A N B$ decrease, as shown in Figure 7. This may be due to decrease in polymerization per wedge, as shown in
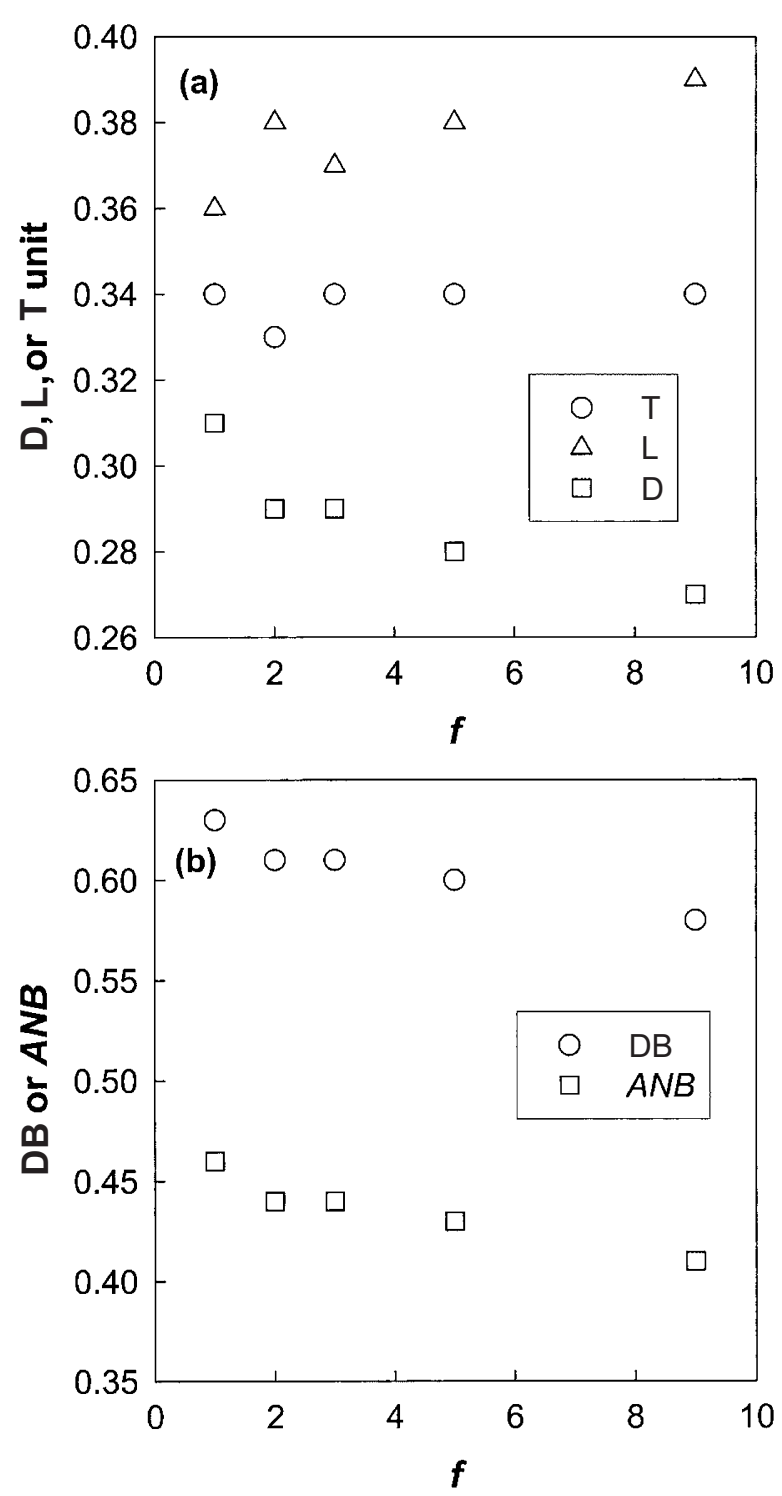

Figure 7. Plots of (a) fraction of dendritic (D), linear (L), and terminal unit (T), and (b) DB and $A N B$ as a function of $f$ with $r=$ 0.01 , when $\mathrm{AB}_{2}$ monomers are polymerized up to $p_{\mathrm{A}}=0.98$ under MBP.

Figure 8. Since each part emanating from one functional group of the core moiety, during polymerization of $\mathrm{AB}_{n}$ type monomers in the presence of $\mathrm{B}_{f}$ type core molecules, can be considered as a dendron or a wedge, the resulting polymer may be regarded as an imperfect dendrimer. As core functionality increases, the number of wedges increases while the degree of polymerization of each wedge decreases (Figure $8 \mathrm{~b}$ ) even though the overall number average degree of polymerization remains nearly constant (Figure 8 a). DB depends upon the conversion, and thus DB can be related to the degree of polymerization. ${ }^{4,9}$

As the concentration of core molecules increases, the number average degree of polymerization decreases, as shown in the first three rows of Table I, because $\mathrm{DP}_{\mathrm{n}}$ is inversely proportional to $r$ as predicted by eq 13. PI 

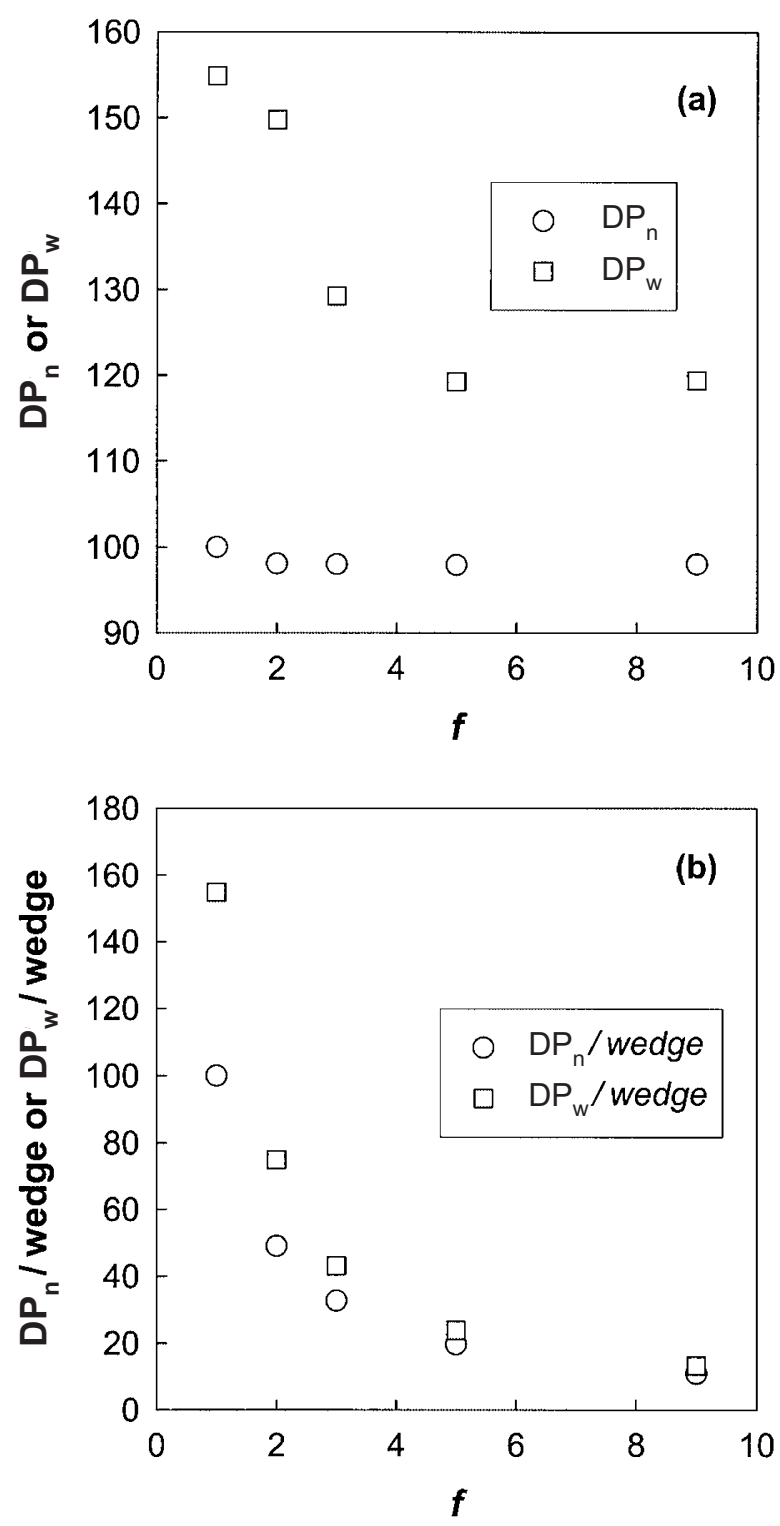

Figure 8. Plots of (a) $\mathrm{DP}_{\mathrm{n}}$ and $\mathrm{DP}_{\mathrm{w}}$ and (b) $\mathrm{DP}_{\mathrm{n}} /$ wedge and $\mathrm{DP}_{\mathrm{w}} /$ wedge as a function of $f$ at $r=0.01$, when $\mathrm{AB}_{2}$ monomers are polymerized up to $p_{\mathrm{A}}=0.98$ under MBP.

Table I. Simulation results under $\mathrm{MBP}$ for $\mathrm{AB}_{2}$ type monomers with $\mathrm{B}_{f}$ type core moieties

\begin{tabular}{rcrcccccc}
\hline$f$ & $r$ & \multicolumn{1}{c}{$\mathrm{DP}_{\mathrm{n}}$} & $P I$ & $\mathrm{~T}$ & $\mathrm{~L}$ & $\mathrm{D}$ & $\mathrm{DB}$ & $A N B$ \\
\hline 1 & 0.01 & 100.00 & 1.55 & 0.34 & 0.36 & 0.31 & 0.63 & 0.46 \\
1 & 0.03 & 33.58 & 1.77 & 0.34 & 0.38 & 0.29 & 0.60 & 0.43 \\
1 & 0.09 & 11.64 & 1.92 & 0.36 & 0.38 & 0.24 & 0.58 & 0.41 \\
3 & 0.03 & 32.90 & 1.38 & 0.35 & 0.40 & 0.25 & 0.55 & 0.38 \\
9 & 0.01 & 98.02 & 1.22 & 0.34 & 0.39 & 0.27 & 0.58 & 0.41 \\
\hline
\end{tabular}

increases and DB decreases with increasing $r$. Increase of $P I$ may be due to increase of the number of polymers in simulation, and decrease of DB may result from decrease in the degree of polymerization.

Simulation results in the last three rows of Table I were obtained at $r \times f=0.09$, which means that there exist an equal number of wedges in the simulation box. At constant wedge concentration, PI becomes lower as
Table II. Simulation results under MBP for $\mathrm{AB}_{n}$ type monomers with $\mathrm{B}_{3}$ type core moieties at $r=0.01$

\begin{tabular}{ccccc}
\hline$n$ & \multicolumn{1}{c}{ DP $_{\mathrm{n}}$} & $P I$ & $\mathrm{DB}$ & $A N B$ \\
\hline 2 & 98.00 & 1.32 & 0.61 & 0.44 \\
4 & 97.96 & 1.47 & 0.49 & 0.57 \\
8 & 116.60 & 2.40 & 0.44 & 0.63 \\
\hline
\end{tabular}

(a)

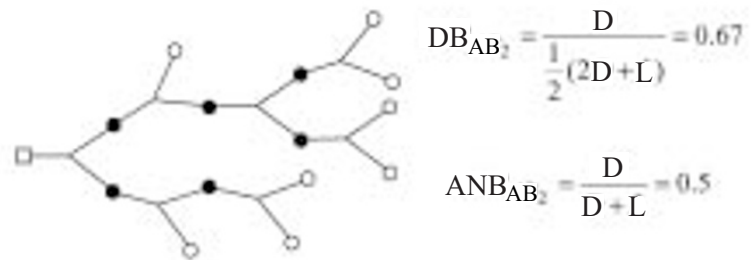

(b)

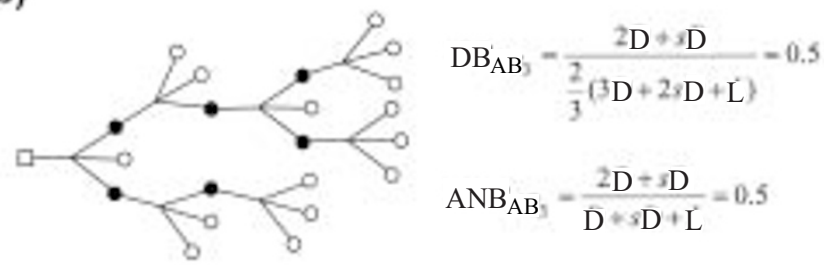

Figure 9. Comparison of degree of branching and average number of branches for two hyperbranched polymers based on (a) $\mathrm{AB}_{2}$ and (b) $\mathrm{AB}_{3}$ monomers. The two polymers have the same branched structure and same degree of polymerization.

core functionality increases whereas DB remains nearly unchanged. It is thus more desirable to use core moieties with high functionality to obtain hyperbranched polymers of lower $P I$ without changing the DB.

Table II lists simulation results for $\mathrm{AB}_{n}$ type monomers at various $n$ in the presence of $\mathrm{B}_{3}$ type core moieties. As B functionality of $\mathrm{AB}_{n}$ type monomer increases, $P I$ and $A N B$ increase whereas $\mathrm{DB}$ decreases. $P I$ was derived as $\left(n-p_{\mathrm{A}}^{2}\right) /\left(n\left(1-p_{\mathrm{A}}\right)\right)$ for homopolycondensation of $\mathrm{AB}_{n}$ type monomers, ${ }^{3}$ and so $P I$ increases with monomer functionality $n$. The same for $P I$ is expected for multibranching polymerization. As the monomer functionality $n$ increases, the probability to form a branched structure becomes larger. However, simulation results show that DB decreases with increasing $n$, as shown in Table II. Hyperbranched polymer thus becomes linear as $n$ increases. This discrepancy between theory and simulation may be explained as follows. DB is defined as the ratio of actual growth direction to maximum possible growth direction. When $A_{2}$ and $A B_{3}$ systems with equal actual growth directions are compared, DBs of two systems become different, because the maximum possible growth direction are different for the two systems (see Figure 9). To overcome such inconsistency, $A N B$ may more reasonably describe the branched structures of hy- 
perbranched polymers. In calculating $A N B$, the nonterminal monomer unit is used as denominator instead of maximum growth directions. As shown in Figure 9, $A N B$ becomes equal for the same branched structure regardless of monomer type. $A N B$ may thus be used as a more universal parameter for describing branched structures of hyperbranched polymers.

\section{CONCLUSIONS}

Kinetic equations describing conversion and degree of polymerization as a function of time for polycondensation (case II) and multibranching polymerization (case III) of $\mathrm{AB}_{n}$ type monomers in the presence of $\mathrm{B}_{f}$ type core molecules were derived. Theoretical equations predict that conversion shows an S-type curve for MBP. The conversion from off-lattice Monte Carlo simulation shows an S-type curve, indicating theory predicts simulated results. Simulation results show that polydispersity, degree of branching and average number of branches decrease with core functionality. With increasing concentration of core molecules, $P I$ increases whereas $\mathrm{DP}_{\mathrm{n}}, \mathrm{DB}$, and $A N B$ decrease. Under constant wedge conditions, $P I$ becomes lower as core functionality increases whereas DB and $A N B$ remain nearly unchanged. Core molecules with high functionality should thus be used to obtain hyperbranched polymers with lower $P I$ without changing DB.

Acknowledgment. The authors thank the Korea Science and Engineering Foundation (KOSEF) for financial support through the Hyperbstructural Organic Materials Research Center (HOMRC).

\section{REFERENCES}

1. M. Johansson, E. Malmström, and A. Hult, Trends in Polym. Sci. , 4, 398 (1996).

2. Y. H. Kim, J. Polym. Sci., Part A: Polym. Chem., 36, 1685 (1998).

3. P. J. Flory, J. Chem. Phys., 74, 2718 (1952).

4. D. Hölter, A. Burgath, and H. Frey, Acta Polym., 48, 30 (1997).

5. H. Frey and D. Hölter, Acta Polym., 50, 67 (1999).

6. E. Malmström, M. Johansson, and A. Hult, Macromolecule, 28, 1698 (1995).

7. D. Yan, Z. Zhou, and A. X. E. Müller, Macromolecules, 32, 245 (1999).

8. D. Yan and Z. Zhou, Macromolecules, 32, 819 (1999).

9. R. Hanselmann, D. Hölter, and H. Frey, Macromolecules, 31, 3790 (1998).

10. M. Suzuki, A. Ii, and T. Saegusa, Macromolecules, 25, 7071 (1992).

11. M. Suzuki, S. Yoshida, K. Shiraga, and T. Saegusa, Macromolecules, 31, 1716 (1998).

12. Y. U. Lee, S. S. Jang, and W. H. Jo, Macromol. Theory Simul., 9, 188 (2000).

13. W. H. Jo and Y. U. Lee, Macromol. Theory Simul., 10, 225 (2001).

14. A. Sunder, R. Hanselmann, H. Frey, and R. Mülhaupt, Macromolecules, 32, 4240 (1999).

15. U. Beginn, C. Drohmann, and M. Möller, Macromolecules, 30, 4112 (1997).

16. D. Yan, Z. Zhou, H. Jiang, and G. Wang, Macromol. Theory Simul., 7, 13 (1998). 